\begin{tabular}{|l|l|l|l|}
\hline Eiszeitalter und Gegenvart & $\mathbf{5 3}$ & $\begin{array}{l}114-123 \\
2 \mathrm{Abb} .\end{array}$ & Hannover 2003 \\
\hline
\end{tabular}

\title{
Nochmals zur Altersstellung des Tranitzer Fluviatils (Anmerkungen zum „Kommentar“ von Lippstreu \& STACKebrandT)
}

\author{
WerNer NoweL ${ }^{*}$
}

Keywords: Middle Pleistocene, Saalian Complex, stratigraphy, till gravel analyses, fluvial sediments, Lower Lusatia, North Germany

Kurzfassung: Eine gründliche Analyse der Lagerungsverhältnisse an und in der Rinne Gosda - Klinge (Tagebau Jänschwalde) beweist die Richtigkeit der von Heı.LWIG (1975) begründeten stratigraphischen Stellung des Tranitzer Fluviatils zwischen den Glazialfolgen Saale (Drenthe) 2 und Warthe 1. Die Flussschotter des Holstein sensu lato und des Tranitzer Fluviatils gehören im Quartär der Niederlausitz unterschiedlichen Stockwerken an. Die drei Tills des Profils Ostrandschlauch sind Bildungen der drei Glazialfolgen des Saale-Komplexes.

[Once more arguments to the stratigraphical position of the Tranitz Fluviatil (remarks to the "commentary" of Lippstreu \& StackebrandT)]

Abstract: The stratigraphical position of the Tranitz Fluviatil, according to HELL.WIG (1975) between the glacial sequences Saalian (Drenthian) 2 and Warthian 1 , is proved as a result of thorough detailed analysis of the stratigraphical conditions of the Gosda - Klinge channel structure (Jänschwalde opencast mine). The fluvial gravel deposits of the Holsteinian sensu lato and the Tranitz Fluviatil are lying in different stratigraphic levels of the Quaternary of Lower Lusatia. The three tills of the old eastern end slope section are three glacial sequences of Saalian Complex.

\footnotetext{
*Anschrift des Verfassers:

Dipl.-Geol. W. Nowel,

Sanzebergstraße 4, D-03042 Cottbus
}

Hauptgegenstand meines Aufsatzes "Zur Korrelation der Glazialfolgen des Saale-Komplexes Nordund Mitteldeutschlands am Beispiel des Tagebaus Jänschwalde in Brandenburg" (Band 52, S. 4783) ist der Versuch einer praktikablen Korrelation zwischen der in Ostdeutschland durch CEPEK begründeten Dreigliederung des Saale-Komplexes (Saale I, II, III) und der gültigen Zweigliederung Nordwestdeutschlands (Drenthe, Warthe) auf Basis der Gedanken zur Zyklentheorie von RichTER (1968) und Ehlers (1992) sowie einer gründlichen Analyse der Literatur zum Problem der umstrittenen Wärmeperiode zwischen Drenthe und Warthe. Die Diskussion neuer Möglichkeiten zum Vergleich der Gliederungen in Polen, Südbrandenburg, Westsachsen, der Altmark, Niedersachsen, Hamburg und Schleswig-Holstein ist das eigentliche Ziel.

Das Pleistozänprofil vom Ostrandschlauch des Tagebaus Jänschwalde dient dabei als regionaler Ausgangspunkt, ist aber für das Gesamtvorhaben nicht entscheidend, sondern könnte durch eine Reihe anderer Niederlausitzer Profile durchaus ersetzt werden (Cepek, Hellawig \& Nowlel 1994).

Gleichwohl ist das Jänschwalder Quartär aufgrund zahlreicher seit 1975 erfolgter Bearbeitungen und geführter Exkursionen von besonderem Interesse. Nicht zu ignorieren ist allerdings auch die Tatsache, dass speziell zum Südrandschlauch des Tagebaus seit 1994 sehr unterschiedliche stratigraphische Interpretationen und daraus abgeleitete Verallgemeinerungen in der Literatur existieren: Sowohl im Heft 1 der „Brandenburgischen Geowiss. Beiträge“ als auch im Tagungsband der 27. DEUQUA-Tagung (EISSMANn \& LitT 1994) stehen die Auffassungen von Cepek ( $\dagger$ ), Heldwig und Nowlil einerseits sowie Lippstreu und Mitarbeitern andererseits kommentarlos nebeneinander und erleichtern dem 
Leser nicht gerade die Orientierung. Bemühungen um eine Annäherung der Standpunkte waren 1992/ 93 im Vorfeld der DEUQUA-Tagung erfolglos geblieben. Deshalb konzentrierten wir uns seitens der LAUBAG bei den Exkursionsführungen für die 27. DEUQUA-Tagung (in EISSMANN \& LiTT I994: I52I68) und den XIV. INQUA-Kongress (in SCHIRmER 1995: 366-372) hauptsächlich auf das instruktivere Profil des Ostrandschlauchs, während Lippstreu (in EISSMANN \& LitT 1994: 168-189) unsere und seine Böschungskartierungen im Südrandschlauch präsentierte.

Quartärgeologisch bildet das Gebiet Dubrau - Klinge - Gosda - Mulknitz (detaillierte Übersichtskarte in Abb. 1) durchaus eine Einheit, und so gibt es rein lithologisch betrachtet zwischen den von mir publizierten geologischen Schnitten 1-3 (NowEL in Eissmann \& LitT 1994: 156, Schnitt 3 siehe auch Abb. 2 meines eingangs genannten Aufsatzes) und dem Kartierungsprofil von Lippstreu et al. (1994: 16a sowie in EISSMANN \& LiTT 1994: 168a) kaum Differenzen; ernsthaften Widerspruch fordern allerdings die konträren stratigraphischen Interpretationen heraus. Auf Seite 6 (und mit Bezugnahme auf ERD auch Seite 26) meines Aufsatzes sind in gebotener Kürze die Fakten und Argumente genannt, die für eine Beibehaltung des von HeLtwig (1975) begründeten Intra-Saale-Alters des Tranitzer Fluviatils und gegen eine von Lippstreu et al. (1994) geforderte Zurückstufung in die Zeit vor der 1. Saale- (Drenthe-) Glaziation sprechen. Auf eine ausführliche Diskussion musste aus Platzgründen verzichtet werden. Nach den erneuten Einwänden durch Lippstreu (in Reissmann \& Böse 2002: 45) und dem „Kommentar" von Lippstreu \& STACKEBRANDT (2003) sind nun etwas detailliertere Anmerkungen unerlässlich.

\section{$1 \mathrm{Zu}$ den Lagerungsverhältnissen in der Gosda - Klinger Rinne}

Die Rinne Gosda - Klinge im Tagebaufeld Jänschwalde ist die größte im Regelbetrieb mit einem Förderbrückenverband F 60 erfolgreich überbaggerte Störungsstruktur im Niederlausitzer Revier. Nach einer Verkürzung der Strosse und Umfahrung des flözfreien Bereichs nördlich der Ortschaft Gosda im Jahre 1986 entstand das 1,3 km lange, die Rinnenstruktur in spitzem Winkel schneidende Ostböschungssystem des Südrandschlauchs (siehe Abb. 1), das wir bereits im Juli 1986 mit einer Exkursion des AK Quartärgeologie der GGW besuchen konnten (Nowel et al. 1986). Besichtigt wurden damals im Grubenbetrieb des Tagebaus (am Punkt 5 in Abb. 1):

- die Viviparus diluvianus KunTH führenden Kiessande, die Heliwig (1975: 1087) an Bohrgut aus der Bohrung 1422/74 als Flussschotter der Lausitzer Neiße aus dem Zeitraum Elster-Kataglazial bis Saale-Anaglazial („Holstein sensu lato“) bestimmt hatte,

- die überlagernde Grundmoräne, in der ebenfalls Exemplare von Viviparus aufgenommen waren und

- die verschiedensten glazigenen Störungsformen im Tertiär der Rinnenflanken.

Seit den Darstellungen von KüHNER (in Nowel et al. 1986: 43) ging man - in Anlehnung an die Geschiebeanalyse von HeLLwig am Bohrgut der Bhg. 1422/74 und die zahlreichen Untersuchungen von CEPEK (1985, unveröff.) aus der benachbarten Dubrau-Bohrauer Rinne - davon aus, dass es sich bei der gesamten Geschiebemergelfüllung der Rinne um Saale I-Grundmoräne handelt, der Saale II-Vorstoßbänderschluff und Reste des Tranitzer Fluviatils auflagern. KüHNER et al. (1988) stellten bei ihrer gründlichen Dokumentation der Rinnenstruktur zwar eine Zweiteilung des Geschiebemergels fest, deuteten diese aber als unterschiedliche Fazies des gSI. Diese Interpretation wurde von NowEL. \& Cepek (1988), Cepek \& Nowel (1991) und auch noch von KÜHNER (in Nowel et al. 1991: 134) kritiklos übernommen.

Für die Exkursion 4/1 der 38. Jahrestagung der GGW 1991 fertigte ich den Schnitt 4 an (Abb. 2), der in der damaligen Fassung nach SE Anschluss an den Saale I-Till der Dubrauer Rinne hatte, der durch CEPEK (1985, 


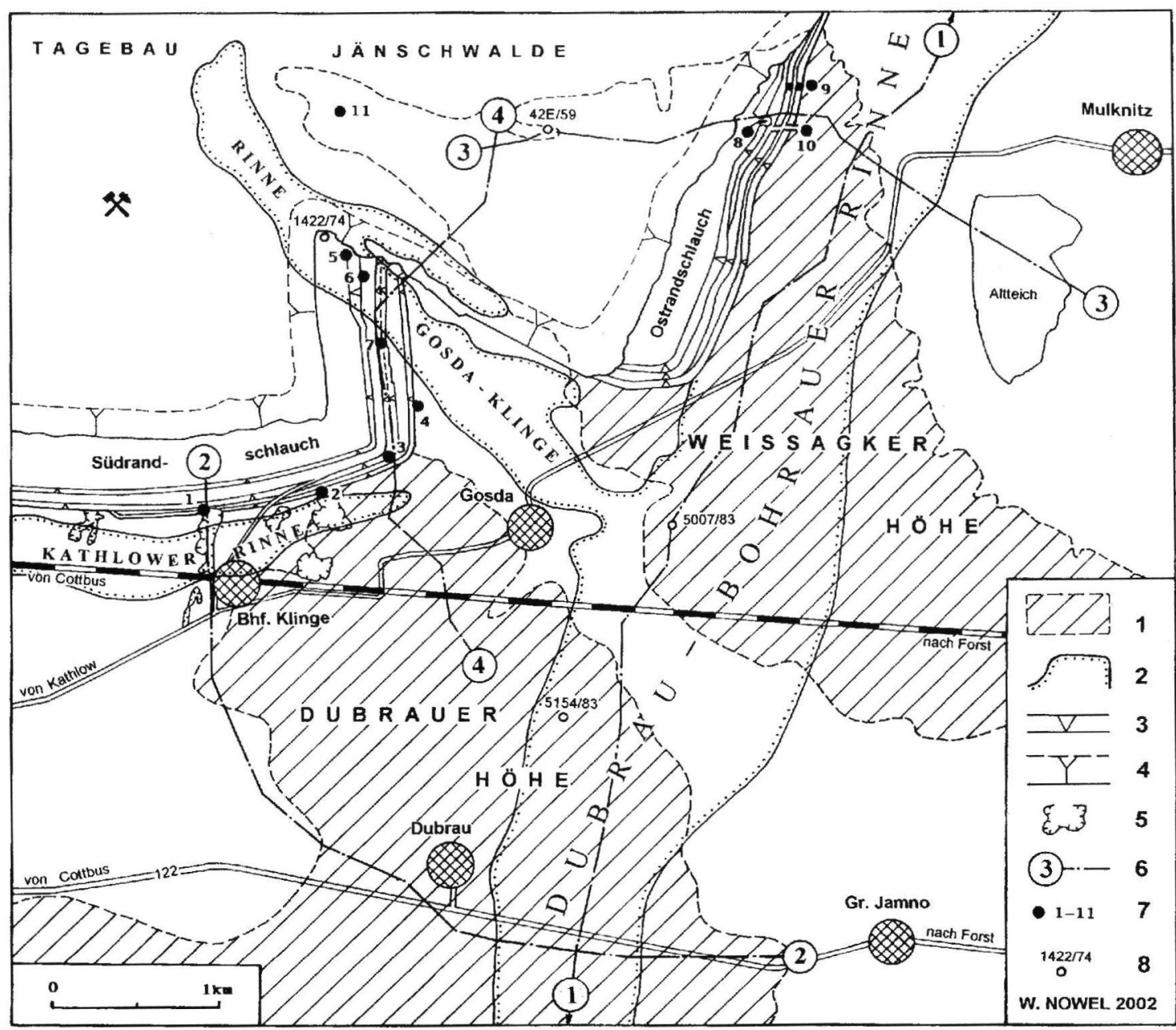

Abb. 1: Übersichtskarte des Gebietes Dubrau - Klinge - Gosda - Mulknitz mit dem Süd- und Ostrandschlauch des Tagebaus Jänschwalde

1 - wartheglaziale Hochflächen; 2 - Kohlefeldergrenzen des 2. Lausitzer Flözhorizontes (Punkte in Richtung der pleistozänen Rinnen); 3 - Abraumbaggerböschungen; 4 - Kippenböschungen; 5 - ehem. Tongruben; 6 - Spurlinien geologischer Schnitte; 7 - spezielle Aufschlusspunkte; 8 - im Text genannte besondere Bohrungen.

\section{Erläuterung zu den Aufschlusspunkten:}

1) Eem-Grabung des Museums der Natur und Umwelt Cottbus in der ehem. Älteren Dominialgrube (R. \& U. Striegler in Nowel et al. 1986: 39, in Eissmann \& Litt 1994: I66, in Reissmann \& Böse 2002: 42) und Tranitzer Fluviatil mit borealen Mudden an der 1. Abraumböschung des Tagebaus ( NowEl et al. 1986, 1991, Nowel \& Ceper 1988, Cepek \& Nowel 1991, Erd in Nowel et al. 1986: 41, Erd 1994a: 38, Cepek et al. sowie Lippstreu et al. in Eismann \& Litt 1994: 167, 183).

2) Grundmoränen sowie Tranitzer Fluviatil an der 1. Abraumböschung unterhalb der ehem. Zweigschen Tongrube (Nowel et al. 1991; Lippstreu et al. in Eismann \& LitT 1994: 185).

3) Referenzprofil für das „Klinger Fluviatil“ mit 6 Mudde-Horizonten an der 2. Abraumböschung (Nowel \& Ceper 1988, CepeK \& Nowel 1991, Nowfi. et al. 1991) und Fundstelle eines Stammes von Quercus sp. in den 
Kiesen des Tranitzer Fluviatils an der 1. Abraumböschung (Lippstreu et al. in Eismann \& LitT 1994:186).

4) nach NE einfallender Geschiebemergel an der 1. Abraumböschung.

5) fluviatile Kiessande des Holstein sensu lato mit Viviparus diluvianus KunTH und Saale I-Till im Niveau der Grube.

6) Saale II-Till im Niveau der 4. Abraumböschung.

7) Saale III- (Warthe 1-) Till an der 3. Abraumböschung.

8) Fundstelle von Viviparus diluvianus Kunth im Niveau der 3. Abraumböschung im Ostrandschlauch.

9) Probenentnahmestellen von Heliwig im Jahre 1993 (Analysen in Eismann \& LitT 1994: 160; in Schirmer I995: 370 und in Nowel 1996: 67).

10) bei den Exkursionen am 16.9.1994, 11.8.1995 und 11.6.1999 vorgeführtes Profil im Saale-Komplex des Ostrandschlauchs (Heldwig, Kühner \& Nowel in Eissmann \& Litt 1994: 164; in Schirmer 1995: 371 und Nowel 1996: 64).

11) ehem. Kiesgrube Weissagk (Heldwig 1975b, in Cepek, Heldwig \& Nowel 1994: 58).

Fig. 1: General map around the villages Dubrau - Klinge - Gosda - Mulknitz including the Southern and Eastern end slopes of the Jänschwalde opencast mine

1 - Warthian ground moraine plateaus; 2 - borderlines of lignite deposits of the 2nd Lusatian Seam Horizon (points lateral of the deep Pleistocene erosion channel structures); 3 - overburden dedger slopes; 4 - slopes of dump; 5 - abandoned clay pits; 6 - lines of geological profile sections; 7 - special outcrop points; 8 - specific boreholes, which are called in text.

\section{Explanations to the special outcrop points:}

1) Eemian outcrop pit of the Natural Science Museum of Cottbus in the former oldest Dominial clay pit (R. \& U. Striegler by Nowel et al. 1986: 39; by Eissmann \& Litt 1994: 166; by Reissmann \& Böse 2002 : 42) and stratigraphic sequence of the Tranitz Fluviatil including boreal muds at the 1 th overburden dredger slope of the opencast mine (Nowel et al. 1986, 1991; Nowel \& Cepek 1988; Cepek \& Nowel 1991; ErD by Nowel et al. 1986: 41, Erd 1994a: 38; Cepek et al. as well as Lippstreu et al. by Eissmann \& LitT 1994: 167, 183).

2) Tills and Tranitz Fluviatil at the 1 th overburden dredger slope below the former ZwEig clay pit (NowEL et al. 1991; Liptstreu et al. by Eissmann \& LitT 1994: 185).

3) reference profile of the "Klinge Fluviatil" including 6 mud horizons at the 2 nd overburden dredger slope (Nowel \& Cepek 1988; Cepek \& Nowel 1991; Nowel et al. 1991) and finding locality of a trunk of Quercus sp. in gravels of the Tranitz Fluviatil at the 1th overburden dredger slope (Lippstreu et al. by EISSMANN \& LitT 1994: 186).

4) northeastwards dipping till at the 1 th overburden dredger slope.

5) fluviale gravel sands of the Holsteinian s. 1. including Viviparus diluvianus KUNTH and Saalian I till outcroping in the level of lignite seam mining.

6) Saalian II till at the 4 th overburden dredger slope .

7) Saalian III (Warthian 1) till at the 3th overburden dredger slope.

8) finding locality of Viviparus diluvianus KunTH in level of the 3th overburden dredger slope inside the old Eastern end slope.

9) sampling locality by HeLLivig 1993 (analyses by EISSMANn \& Litt 1994: 160; by Schirmer 1995: 370 and by Nowel 1996: 67).

10) geological outcrop profile of the Saalian Complex at the old Eastern end slope, which are presented during the excursions on 1994-9-16, 1995-8-11 and 1999-6-11 (Helliwig, Kühner \& Nowel by Eissmann \& LitT 1994: 164; by SChirmer 1995: 371; by Nowel 1996: 64).

11) former gravel pit of Weissagk (Heldwig 1975b; by Cepek, Heldivig \& Nowel 1994: 58). 
unveröff.) an 7 Proben aus der Bhg. 5154/83 geschiebeanalytisch als solcher ausgewiesen ist. Bei der Böschungskartierung zur Präzisierung des Schnittes im Bereich des offenen Tagebaus stellte ich an der 1. Abraumböschung (4 in Abb. 1, ca. $100 \mathrm{~m}$ östlich der Bhg. 1480/74) völlig unerwartet fest, dass der Geschiebemergel aus der Gosda-Klinger Rinne in einer schmalen Zunge bis wenige Meter unter Gelände aufragt. Er wird hier erosionsdiskordant nur von geringmächtigem Beckensand, wahrscheinlichem Eem und deluvial-fluviatilen Sanden

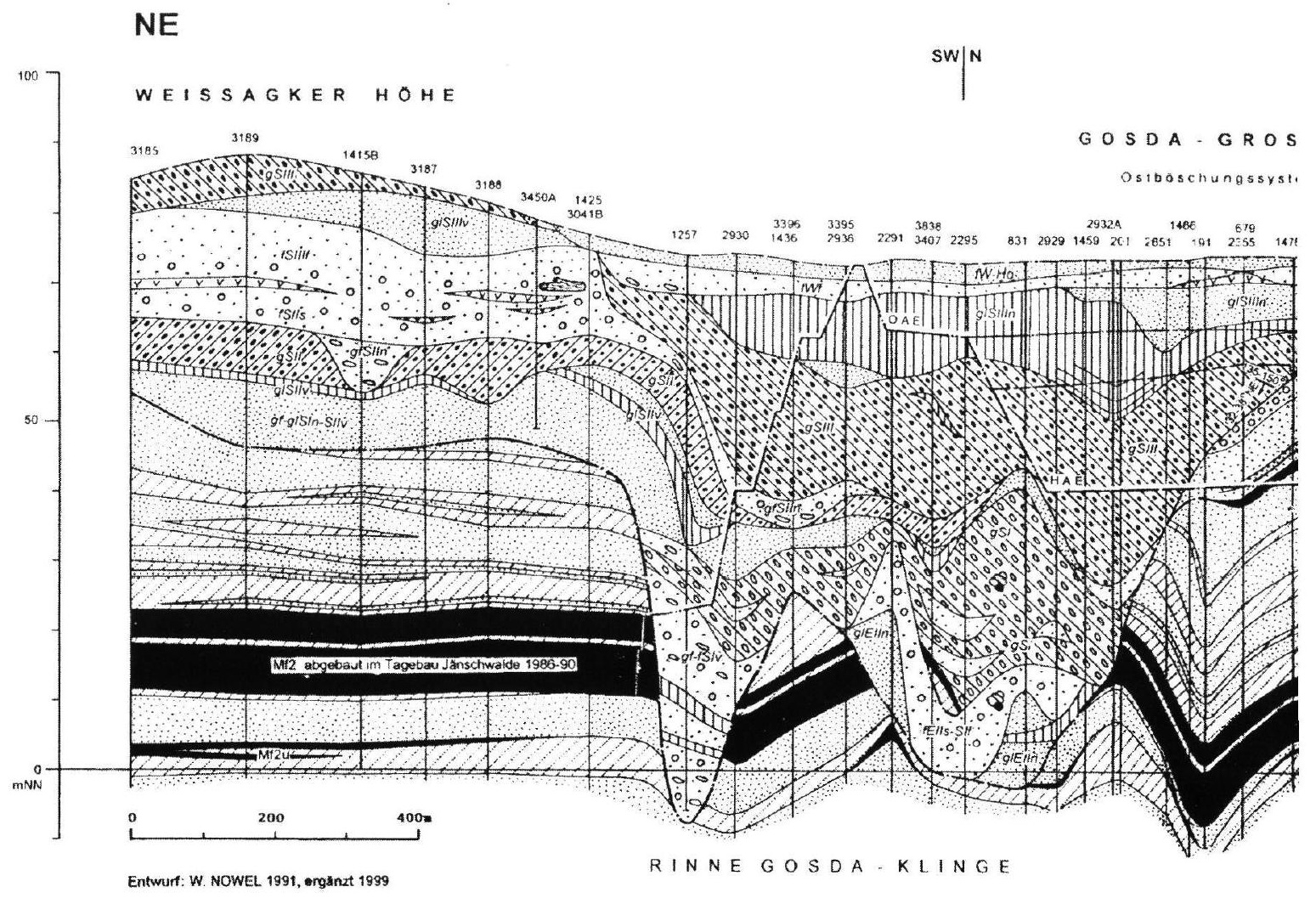

Abb. 2: Geologischer Schnitt 4 von der Weissagker Höhe zur Dubrauer Höhe durch die Rinne Gosda - Klinge und die Kathlower Rinne (Darstellung 10fach überhöht).

1-3 Tertiär, dabei: 1 - Braunkohle (Mf2 = miozäner 2. Lausitzer Flözhorizont, Mf2u = Unterbegleiter des Mf2); 2 - Schluff und Ton; 3 - Feinsand; 4 - Grenze Tertiär/ Quartär; 5-17 Quartär, dabei: 5 - Grundmoräne Elster II; 6 - Grundmoräne Saale I (Drenthe 1); 7 - Grundmoräne Saale II (Drenthe 2); 8 - Grundmoräne Saale III (Warthe 1); 9 - Steine; 10 - Kies und Kiessand; 11 - Mittel- und Grobsand; 12 - Feinsand; 13 - Ton und Schluff einschl. Bänderton; 14 - Ton- und Schluffmudden im „Klinger Fluviatil“ (Ziffern 1-6 = Muddehorizonte bei Nowel \& CEPEK 1988 u.a.); 15 - Ton-, Schluff- und Torfmudde im Tranitzer Fluviatil und im wahrscheinlichen Eem; 16 - Viviparus diluvianus KunTH (die Funde stammen nicht aus den Bohrungen, sondern aus dem Tagebauaufschluss beim Punkt 5 in Abb. 1); 17 - Funde von Treibholz im Tranitzer Fluviatil; 18 - technologische Grenzen im Böschungssystem des Tagebaus (HAE = Hauptarbeitsebene, OAE = obere Arbeitsebene des ehem. Förderbrückenverbandes). Die Einschreibungen in den Schichten entsprechen denen der Lithofazieskarten Quartär 1:50 000 (Erläuterungen bei CEPEK [†] 1999, siehe auch Nowel 2003: Abb. 2 u. 3). 
des Gosda-Groß Jamnoer Tals überdeckt und überlagert seinerseits diskordant die typischen Kiessande des Tranitzer Fluviatils.

Die aus dem Gesamtbild des Schnittes 4 (Abb. 2) ersichtlichen Lagerungsverhältnisse verdeutlichen, dass das Tranitzer Fluviatil zusammen mit den unterlagernden älteren quartären und tertiären Schichten postgenetisch glazigen gestaucht und eingemuldet worden ist. Etwa im Bereich der Bhg. 191 wird es (einschließlich der Feinsande und Mudden des unterlagernden „Klinger Fluviatils") im Niveau der 3. Abraum-
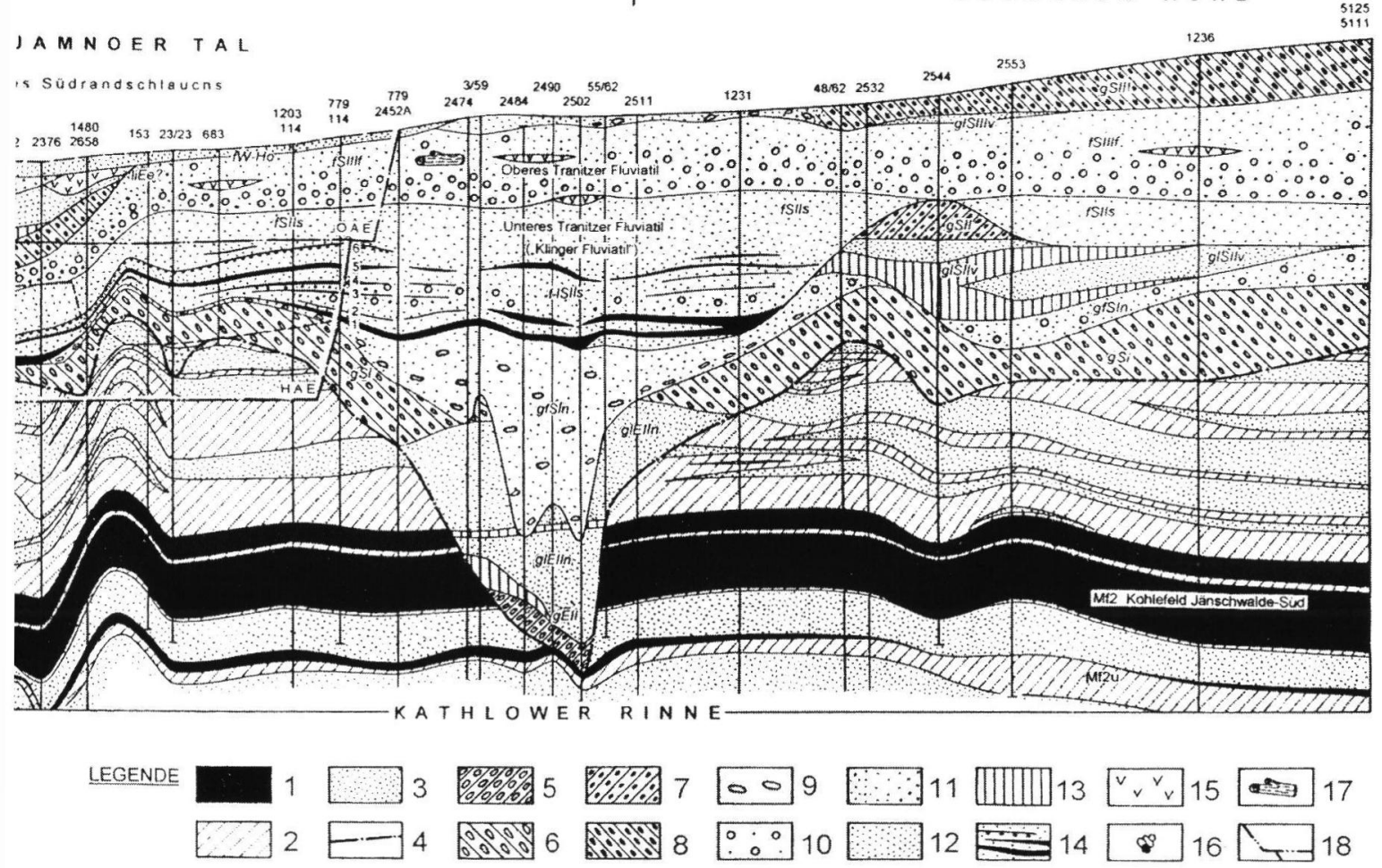

Fig. 2: Geological profile section 4 , going from the ground moraine plateau of Weissagk to the ground moraine plateau of Dubrau cutting through the Pleistocene erosion channel structures of Gosda - Klinge and Kathlow (Vertical exaggeration 10:1)

1-3 Tertiary, consist of: 1 - Lignite (Mf2 = Miocene 2nd Lusatian Seam Horizon, Mf2 $\mathrm{u}=$ under attendant of them); 2 - silt and clay; 3 - fine sand; 4 - Tertiary/ Quaternary boundary line; 5-17 Quaternary, consist of: 5 - Elsterian II till; 6 - Saalian I (Drenthian 1) till; 7 - Saalian II (Drenthian 2) till; 8 - Saalian III (Warthian 1) till; 9 - Scandinavian boulders; 10 - gravel and gravel sands; 11 - middle and coarse sand; 12 - fine sand; 13 - clay and silt including banded clay; 14 - clayish and silty muds of "Klinge Fluviatil" (numerals 1-6 = mud horizons by Nowel \& CEPEK 1988 a.s.o.); 15 - clayish, silty and peaty muds of Tranitz Fluviatil and, probable, Eemian; 16 - Viviparus diluvianus KUNTH (the presented samples not are findet in the bore-holes, but at the opencast mine outcrop see point 5 in fig. 1); 17 - samples of drift wood of Tranitz Fluviatil; 18 - technological borderlines of slope system of the opencast mine (HAE = main working level, $\mathrm{OAE}=$ upper working level of the overburden conveying bridge, which has been working here). The symbols which are writed in the geological bed signatures are according to the Lithofazies Maps of Quaternary scale 1:50 000 (comment by CEPEK [†] 1999, see also NOWEL 2003: fig. 2 and 3). 
böschung (oberhalb der ehem. HAE) exarativ abgeschnitten und grenzt diskordant gegen den Geschiebemergel der Rinne.

Während der Exkursion am 21.10.1991 versuchten wir zunächst, diese Lagerungsverhältnisse durch „saale III-zeitliche Überschiebung des SI-Geschiebemergels" zu erklären (NowEL et al. 1991: 134). Nach Konsultation von D. Hellwwig setzte sich noch im gleichen Jahr die Erkenntnis durch, dass zwischen dem Grundmoränenmaterial in der Gosda - Klinger Rinne und den saale III- (warthe 1-) zeitlichen Grundmoränen auf der Weissagker und Dubrauer Höhe offenbar trotz fehlender direkter Verbindung ein genetischer Zusammenhang besteht. Nach Verlängerung des Schnittes 4 in nordöstlicher Richtung bis zur Weissagker Höhe (Anschluss an den Schnitt 3) konnte im Bereich der Kernbohrungen 1425/76 und 3041B/81 die „Aufschleppung" der Grundmoräne auch am nordöstlichen Rinnenrand nachgewiesen werden. Beide Bohrungen sind nur wenige Meter voneinander entfernt (deshalb im Schnitt 4 nicht getrennt dargestellt): Während die Erstere das Tranitzer Fluviatil mit Treibholzresten in normaler Ausbildung durchteufte, wies die Letztere im gleichen Niveau sandig-schluffigen Geschiebemergel nach.

Auch für Lippstreu et al. (1994: 25) „liegt der Schluss sehr nahe", dass die Grundmoränen der Hochflächen und der Rinne zu konnektieren sind, sie sehen aber in dem Grundmoränenmaterial der Rinne "die dolomitreiche Fazies der Älteren Saalegrundmoräne (qsD)" und darin den von Lippstreu \& Stackebrandt (2003) wiederholten Hauptbeweis für ein prädrenthezeitliches Alter des Tranitzer Fluviatils. Noch in Lippstreu et al. (1993, unveröff.) hatten sie auf der Grundlage des gleichen Kartierungsschnittes und der gleichen Geschiebeanalysen eine Einstufung als „Grundmoräne des Warthestadials (qsWA)" vorgenommen!

Nach neuen Geschiebeanalysen von HeLLwIG an kalkhaltigen Proben konnten CEpek, HeliWIG \& Nowel (1994: 73) konstatieren, dass es sich bei dem Grundmoränenmaterial am Oststoß des Südrandschlauchs weder um einen aufgeschleppten gSI-Horizont (wie Nowel et al. 1991 ohne Geschiebeanalysen vermuteten) noch um die von Lippstreu gedeutete Variante handelt: „Hier liegt eine exarativ geschaffene Einmuldung von Saale III-Grundmoräne mit Schollen von glazilimnischen Sanden, glazilimnischen Schluffen und SII-Geschiebemergel vor. Diese Aussage war erst möglich, nachdem normal kalkhaltige Geschiebemergelpakete für die Untersuchung zugänglich waren".

In der endgültigen Fassung des Schnittes 4 (Abb. 2), die ich erst zu meinem Berliner Vortrag 1999 fertigstellte, ist auf der Grundlage unserer Geschiebeanalysen und Böschungskartierungen sowie der Auswertung zahlreicher Bohrergebnisse eine gewisse Dreigliederung des Geschiebemergelpaketes in der Gosda-Klinger Rinne möglich:

- Der graue bis bräunlichgraue und meist sehr feste Geschiebemergel vom Typ SI (= Drenthe 1 sensu NOWEL) beschränkt sich meist auf den untersten Bereich der Rinne und erreicht wohl nur selten das Niveau der HAE. Die Probennahme erfolgte am Punkt 5 in Abb. 1 im stratigraphischen Hangenden der Flussschotter des Holstein sensu lato. Dieser Geschiebemergel ist zusammen mit den unterlagernden quartären und tertiären Schichten stark glazigen deformiert und m.E. mit dem weiter südlich oberhalb der HAE einsetzenden gSI zu konnektieren.

- Am Punkt 6 in Abb. 1 in einer eingeschnittenen Schlucht wenig unterhalb der HAE konnte ein dunkelgrauer bis schwarzer, glazigen schräg gestellter Geschiebemergel mit Resten von Bänderschluff an der Basis lokalisiert und beprobt werden. Es handelt sich um einen typischen Geschiebemergel des Typs SII (= Drenthe 2 sensu NowEL). Die Lagerungsverhältnisse entsprechen etwa dem Bereich der Bohrungen 3838 und 3407 im Schnitt 4, sehr wahrscheinlich ist eine direkte Verbindung zu dem nach NE bis zum 
Ostrandschlauch flächenhaft aushaltenden gSII gegeben.

- An einer Klippe im oberen Teil der 3. Abraumböschung (Punkt 7 in Abb. 1) wurde der mächtige graubraune bis dunkelbraune und sandige obere Geschiebemergel beprobt. Das Geschiebespektrum entspricht dem Typ SIII (= Warthe 1 sensu NoweL), auch wenn die NK/D-Werte mit 18,5 - 32.1 niedriger sind als in den Analysen vom Ostrandschlauch. Ursache dafür sind aus dem exarierten SII-Till aufgenommene erhöhte Dolomitanteile.

Ein Vergleich mit den Analysenergebnissen von Lippstreu et al. (1994) bestätigt unsere Befunde. Die auf der Lippstreuschen Abb. 2 präsentierten Durchschnittswerte liefern zwar kein eindeutiges Bild, die Einzelwerte seiner Tabelle 2 (Seite 29) jedoch lassen eine differenzierte Zusammensetzung erkennen:

- Die Proben 1 bis 7, bei bestimmten Quotienten bis Probe 11, belegen einen Till vom Typ SIII mit NK/D meist $>18, \mathrm{Ffr} / \mathrm{PK}$ meist $>1$, $\mathrm{PK}+\mathrm{D} / \mathrm{S}$ meist $<5$ und NK/PK größer oder zumindest nahe 1,4. Abweichende Werte belegen aufgenommenes SII-Material. Beim Quotienten $\mathrm{PKgr} / \mathrm{r}+\mathrm{sz}$ sprechen nur die Proben 1, 6 und 7 für SIII, alle anderen sind deutlich $>10$ (= SII). - Die Proben 8 (z.T. 12) bis 15 und 17 bis 18 belegen einen Till vom Typ SII mit NK/D $<18$ (meist < 13), $\mathrm{Ffr} / \mathrm{PK}$ meist $<1, \mathrm{PK}+\mathrm{D} / \mathrm{S}$ meist $>$ 5 und NK/PK überwiegend $<1,4$.

- Bei der Probe 16 mit NK/D = 23,25, Ffr/PK = $0,13, \mathrm{PK}+\mathrm{D} / \mathrm{S}=3,11, \mathrm{NK} / \mathrm{PK}=1,69$ und $50 \%$ Quarz und Südlichen kann es sich um den basalen Saale I-Till handeln.

Fazit: Das wesentlichste Argument von LippSTREu \& Stackebrandt (2003) gegen ein Intra-Saale-Alter des Tranitzer Fluviatils ist damit entkräftet ebenso wie der aus regionaler Sicht unverständliche Versuch, die Grundmoränen auf den warthezeitlichen Weissagker und Dubrauer Hochflächen dem älteren Saale (qsD) zuzuordnen.

\section{Zum Fluviatil} des Holstein sensu lato

Die zwischen 0 und $20 \mathrm{~m} \mathrm{NN}$ im Grubenbetrieb Jänschwalde (5 in Abb. 1) freigelegten Kiessande des Holstein sensu lato sind autochthone Bildungen. Sie werden von elsterkaltzeitlichen Sedimenten unter- und von Saale I-Till überlagert (KÜHNER in Nowel et al. 1986, KÜHNER et al. 1988 sowie zahlreiche unveröff. Unterlagen der Betriebserkundung und Bergbauprojektierung), zum Tranitzer Fluviatil besteht auch bei glazigen gestörter Lagerung keinerlei Verbindung. Über gleichartige Kiese im Aufschlussraum des Tagebaus Jänschwalde bei Grötsch und im Aufschlussraum des Tagebaus Cottbus-Nord (Nowel 1983: 21) zeichnen sie in Relikten einen Flusslauf nach, der südöstlich Peitz in den großen Sedimentationsraum des von HeL.Lwig ausgegrenzten „Elster II-Kataglazial bis Saale I-Anaglazial - Fluviatils" einmündet (CEPEK, Heldwig \& Nower 1994: Abb. 3). Maßgeblich für die Bestimmung des Holstein-Niveaus ist hier das in Abb. 3 meines Aufsatzes zur Argumentation herangezogene Vorkommen der Bhg. 1504/71 (Mudde bei 20,2 - 24,2 m NN; ERD 1994a), weiter entfernt liegen in gleicher Fazies die Profile Groß Drewitz I/60 (ERD in Hannemann 1964, Hellwig \& Krueger 1983) und Butzen (Sonntag 1987, Erd pers. Mitt.).

Das von Lippstreu \& Stackebrandt (2003) angeführte Holstein der Bhg. 3919A3/89 bei Göllnitz-Lug (Gesamtsituation in Schroeder \& Nowei 1995: Abb. 2-5) mit seiner Oberkante bei $74 \mathrm{~m} \mathrm{NN}$ ist für einen direkten Vergleich mit dem Tranitzer Fluviatil bei Klinge nicht geeignet, denn es ist in südwestlicher Richtung $46 \mathrm{~km}$ entfernt. Die überlagernden frühsaalezeitlichen Kiessande gehören zum Flusssystem der Schwarzen Elster. Da alle Flüsse sich auf ein vergleichbares Abflussniveau beziehen, ermitteln wir das theorethische Flussgefälle von der 3919A3/89 bis zur 1504/71 bei Maust. Es ergibt bei $40 \mathrm{~km}$ Entfernung und $50 \mathrm{~m}$ Höhenunterschied einen ganz normalen Wert von 1,25 
$\mathrm{m} / \mathrm{km}$. Rechnet man von Maust weiter nach Groß Drewitz, das schon in das Fünfeichener Becken überleitet, so ergibt sich bei 24 km Entfernung und $24 \mathrm{~m}$ Höhenunterschied ein Wert von $1 \mathrm{~m} / \mathrm{km}$.

Das von Lippstreu \& Stackebrandt (2003) diskutierte Beispiel Müllrose ist wenig geeignet, befindet es sich doch ebenso wie die oberflächennahen Holstein-Vorkommen von Eisenhüttenstadt in einem glazigen gestörten Gebiet. Von größerem Interesse ist in diesem Zusammenhang die fast in Vergessenheit geratene Tatsache, dass zur Exkursion des AK Braunkohle der GGW am 13.5.1988 am nordöstlichen Strossenende des Tagebaus Welzow-Süd (bei der ehem. Ortschaft Groß Buckow) im Niveau der HAE fluviatile Kiessande des Holstein sensu lato mit Muddelagen, Treibholz und mehreren Exemplaren von Viviparus diluvianus KUNTH aufgeschlossen waren, die aus Zeitmangel leider keine spezielle Bearbeitung erfahren haben. Sie gehören zum Flusssystem der Spree und wurden durch HeLlwig in den Lithofazieskarten Quartär 1:50 000 (Blätter 2469 Hoyerswerda und 2369 Cottbus) in ihrer Verbreitung bis in den Raum Cottbus - Peitz genau ausgegrenzt (CEPEK, HeLlwig \& Nowel 1994: Abb. 3). Verfolgt man die natürliche Flussschüttung westlich an Cottbus vorbei bis zur Bhg. 1504/71 bei Maust, so ergibt sich bei ca. $25 \mathrm{~km}$ Entfernung und 30 $\mathrm{m}$ Höhenunterschied ein wiederum ganz normales Flussgefälle von $1,2 \mathrm{~m} / \mathrm{km}$.

Fazit: Die Flussschotter des Holstein s. I. und des Tranitzer Fluviatils gehören im Quartär der Niederlausitz unterschiedlichen Stockwerken an.

\section{$3 \mathrm{Zu}$ den absoluten Altersdaten}

Die TL-Datierung von $247+43-39$ ka BP für eine Schluffschicht im Tranitzer Fluviatil durch KRbetschek (in EIsSMann \& LitT 1994: 295) kann leider nicht zur Klärung des Altersstreits beitragen, da die Kontrahenten unterschiedlichen Modellvorstellungen anhängen.
Lippstreu \& Stackebrandt (2003) vertreten das Eissmannsche Modell einer monoglazialen Entwicklung von Drenthe und Warthe innerhalb der ${ }^{18} \mathrm{O}$-Stufe 6 und halten die o.g. Daten für einen Beweis prädrenthezeitlichen Alters. Nach den Argumenten im Abschnitt 4.7 meines Aufsatzes wird der drenthezeitliche Vereisungszyklus der ${ }^{18} \mathrm{O}$-Stufe 8 und der warthezeitliche Vereisungszyklus der ${ }^{18} \mathrm{O}$-Stufe 6 zugeordnet; so stehen die o.g. Daten für eine Drenthe/ Warthe-Wärmeperiode in Stufe 7. In gleicher Weise subjektiv sind die Lippstreuschen Argumente zur Bewertung warmzeitlicher Verwitterungserscheinungen. Leider war es 1999 nicht gelungen, die von Herrn Professor K.-D. JÄGER, Halle, vorgeschlagenen engmaschigen TL-Datierungen am vorbereiteten Profil Ostrandschlauch durch Herrn KRBETSCHEK zu realisieren, das ist sehr bedauerlich.

\section{Zu den Viviparus - Funden im Ostrandschlauch}

Die Funde wurden in der großen Schlucht in der 3. Abraumböschung nahe des bei den Exkursionen vorgeführten Profils gemacht (8 in Abb. 1), an der Herkunft aus dem anstehenden Saale I-Till gibt es keinen Zweifel. Auch mit Bezugnahme auf das unter 1. und 2. Gesagte wiederhole ich die Feststellung, dass es sich bei den drei Tills des Profils Ostrandschlauch Jänschwalde tatsächlich um Bildungen der drei Glazialfolgen Saale I, Saale II und Saale III im Sinne von CEPEK handelt.

\section{Schriftenverzeichnis}

(Nur die bei Nowel (2003) sowie Lippstreu \& STACKEBRANDT (2003) nicht genannten Arbeiten).

Cepek, A. G. (1985): Bericht über Stratigraphie und Genese der Schichtenfolge des Quar- 
tärs im Braunkohlen-Erkundungsobjekt Jänschwalde-Süd. - Zentrales Geologisches Institut, 17 S., 3 Anl., Berlin (unveröff.). - (†) (1999): Die Lithofazieskarten Quartär 1:50 000 (LKQ 50) - eine Erläuterung des Kartenkonzepts mit Hinweisen für den Gebrauch. Mit Vorbemerkungen von L. Lippstreu. - Brandenburgische Geowiss. Beitr., 6 (2): 3-38; Kleinmachnow.

Hannemann, M. (1964): Quartärbasis und älteres Quartär in Ostbrandenburg. - Z . angew. Geol., 10: 370-376; Berlin.

Hellwig, D. (1975): Aufschlüsse bei Weissagk. - In: Exkursionsführer zur Jubiläumstagung „100 Jahre Glazialtheorie im Gebiet der skandinavischen Vereisungen", 3. bis 7. November 1975 in Berlin, Ges. Geol. Wiss. DDR u. Geogr. Ges. DDR: 36-45; Berlin (1975b). - \& Krueger, I. (1983): Lithofazieskarte Quartär im Maßstab 1:50 000, Blatt 2270 Wilhelm-Pieck-Stadt Guben. - Zentrales Geologisches Institut; Berlin.

Kühner, R., Kloss, R., Baumann, K. \& MädLER, F. (1988): Stratigraphisch-genetische Erkenntnisse aus der Überbaggerung der Rinne Gosda-Klinge. - Z. angew. Geol., 34 (11): 335-338; Berlin.

Lippstreu, L., Hannemann, M., Hermsdorf, N., Jortzig, H., Sonntag, A. \& Thieke, H. U. (1993): Neue Ergebnisse zur Gliederung der quartären Schichtenfolge des Braunkohlenfeldes Jänschwalde ( Niederlausitz) - ein Beitrag zur Gliederung der Saale-Kaltzeit. Kurzfassg. Manuskript zur Tagung der „Geowissenschaftler in Berlin und Brandenburg e.V." am 24.4.1993; Berlin (unveröff.).

Lippstreu, L. \& Stackebrandt, W. (2003): Jänschwalde und die Gliederung des SaaleKomplexes - ein Kommentar zum Beitrag von Werner Nowel. - Eiszeitalter und Gegenwart, 52: 84-90; Hannover.

Nowel, W., Cepek, A.G., Erd, K., Kühner, R., Striegler, U. \& Striegler, R. (1986): Exkursion II: Schichtenfolge und Lagerungsverhältnisse des Quartärs im Raum
Klinge - Dubrau (Krs. Forst). - Kurzref. u. Exkurs. - F. „25 Jahre AK Quartärgeologie der GGW“: 33-48, 1 Tafelbeil., Ges. Geol. Wiss. DDR; Berlin.

Nowel, W., Striegler, R., Cepek, A.G. \& KüHner, R. (1991): Das Pleistozänprofil von Gosda-Klinge - Dubrau (Braunkohlefeld Jänschwalde) - ein Typusgebiet für den Saale-Komplex der Niederlausitz. - Kurzref. u. Exkurs. - F. zur 38. Jahrestagg. der GGW: 125-134; Berlin.

Reissmann, C. \& Böse, M. [Hrsg.] (2002): Exkursionsführer DEUQUA 2002. - Deutsche Quartärvereinigung e.V.: 170 S.; Berlin, Potsdam.

Schroeder, J.H. \& Nowel, W. [Hrsg.] (1995): Führer zur Geologie von Berlin und Brandenburg, Nr. 3: Lübbenau - Calau. - Selbstverlag der "Geowissenschaftler in Berlin und Brandenburg e.V.“" 284 S.; Berlin.

SonNTAG, A. (1987): Lithofazieskarte Quartär im Maßstab 1:50 000, Blatt 2269 Lieberose. - Zentrales Geologisches Institut; Berlin. 